Nonlinear Processes in Geophysics, 12, 871-876, 2005

SRef-ID: $1607-7946 / \mathrm{npg} / 2005-12-871$

European Geosciences Union

(c) 2005 Author(s). This work is licensed

under a Creative Commons License.

\title{
Stochastic parametric resonance in shear flows
}

\section{F. J. Poulin and M. Scott}

University of Waterloo, Waterloo, Ontario, Canada

Received: 1 August 2005 - Revised: 7 October 2005 - Accepted: 13 October 2005 - Published: 3 November 2005

Part of Special Issue "Turbulent transport in geosciences"

\begin{abstract}
Time-periodic shear flows can give rise to Parametric Instability (PI), as in the case of the Mathieu equation (Stoker, 1950; Nayfeh and Mook, 1995). This mechanism results from a resonance between the oscillatory basic state and waves that are superimposed on it. Farrell and Ioannou (1996a, b) explain that PI occurs because the snapshots of the velocity profile are subject to transient growth. If the flows were purely steady the transient growth would subside and not have any long lasting effect. However, the coupling between transient growth and the time variation of the basic state create PI. Mathematically, transient growth, and therefore PI, are due to the nonorthogonal eigenspace in the linearized system.

Poulin et al. (2003) studied a time-periodic barotropic shear flow that exhibited PI, and thereby produced mixing at the interface between Potential Vorticity (PV) fronts. The instability led to the formation of vortices that were stretched. A later study of an oscillatory current in the Cape Cod Bay illustrated that PI can occur in realistic shear flows (Poulin and Flierl, 2005) ${ }^{1}$. These studies assumed that the basic state was periodic with a constant frequency and amplitude. In this work we study a shear flow similar to that found in Poulin et al. (2003), but now where the magnitude of vorticity is a stochastic variable. We determine that in the case of stochastic shear flows the transient growth of perturbations of the snapshots of the basic state still generate PI.
\end{abstract}

\section{Introduction}

Currents and shear flows are a very important means by which fluids mix physical, biological or chemical properties. Particular velocity profiles with a large enough Reynolds number may become unstable and generate vortices which

\footnotetext{
Correspondence to: F. J. Poulin

(fpoulin@uwaterloo.ca)

${ }^{1}$ Poulin, F. and Flierl, G.: A Barotropic Mode of the Cape Cod Bay, in preparation, 2005.
}

can then transport anomalous properties into the ambient fluid. This process has been frequently observed and studied in both laboratory experiments as well as oceanic observations (Griffiths et al., 1982).

One of the most famous ocean currents that is known to generate vortices due to instability is the Gulf Stream. Many of the analytical studies of the Gulf Stream assume the current is steady and then apply a linear stability analysis to predict the growth rates of the instability and also the length scale of the emerging vortices (or rings). Even though this analysis neglected many aspects of the Gulf Stream the simple model manages to capture the essential physics which is evident by the close comparison between the theory and the observations (Flierl, 1977).

There are numerous other examples in which models of currents, and shear flows in general, assume the basic state to be steady. Even though these models can capture the leading order behaviour they neglect the fact that there is inherent time variation in any physical system. Sometimes the stability of the time-dependent system will be quite similar to the stability of the time-averaged system. However there are other situations where time dependence can give very different results. Some prime examples of this are recent studies of time-periodic baroclinic and barotropic shear flows in Pedlosky and Thomson (2003) and Poulin et al. (2003), respectively. These articles clearly indicate that the time average of a state can have very different stability properties than that of the time-dependent state itself. In particular, in Poulin et al. (2003) an example was given of a shear flow where not only was the time-averaged flow stable, but every snap shot in time was also stable (we use the word stable to indicate that there was no exponential growth). Regardless, the time varying state gives rise to exponential growth that is referred to as parametric instability (PI) or parametric resonance (PR). The simplest example of PI is in the Mathieu equation where the natural frequency of the pendulum is a time-periodic function (Stoker, 1950; Nayfeh and Mook, 1995). In the case of barotropic shear flows we call this process PR since the instability process can be interpreted as a 
resonance between the temporally periodic basic state and two Rossby waves which exist at the interface between regions of constant vorticity. Our model of the Cape Cod Bay in Poulin and Flierl (2005) ${ }^{1}$ demonstrated that parametric instability can occur in time-periodic shear flows as often arise in coastal waters. Unfortunately, the sparsity of the observations prevents us from validating these predictions at this time.

It is natural to ask whether this instability mechanism is dependent upon the basic state being purely periodic or whether this can also arise from a non-periodic timedependent basic state. If it does require that the shear flow is periodic the results are very limited since it is rare, if not impossible, to get a perfectly periodic oscillation. The main point of this article is to consider the other extreme of timedependency, where the shear flow has stochastic variation. Can a stochastic shear flow with stable time-averaged mean give rise to instability in the time-dependent system? This question is much harder to answer since we can no longer use Floquet analysis to analyse the growth rates of the basic state due to the loss of periodicity. Moreover, stochastic differential equations are notoriously difficult to to solve. By using certain approximations we will illustrate how stochastic shear flows can give rise to an instability and we call this phenomenon stochastic parametric resonance. We are unaware of any applications of this theory to shear flows which is the novel aspect of the problem.

\section{Generalised stability analysis}

For the case of a steady shear flow (or in general a steady basic state) one can perturb the basic state and linearise if we assume that the perturbations are small. This allows us to write the governing equations as a linear system of differential equations, in some cases a linear system of ordinary differential equations (ODEs). The general form of this is written below where the perturbation vector is denoted by $\boldsymbol{x}$ and the matrix $\mathbf{A}_{0}$ contains information about the basic state.

$$
\frac{d \boldsymbol{x}}{d t}=\mathbf{A}_{0} \boldsymbol{x}
$$

To determine the stability of a basic state we must compute the eigenvalues and eigenvectors of the matrix $\mathbf{A}_{0}$. This will capture any growth or decay that is exponential. If we want to determine if there is any algebraic growth, as can occur in transient growth, it is necessary to look at a non-normal analysis. The largest singular values of $e^{\mathbf{A}_{0}}$ correspond to the largest transient growth, albeit only temporarily (Farrell and Ioannou, 1996a).

Let us consider a more complex scenario where the basic state is nearly steady but has some slight time variation added on to it. If we assume that the perturbations are small we can obtain the following linearized system of equations

$$
\frac{d \boldsymbol{x}}{d t}=\left[\mathbf{A}_{0}+\epsilon \eta(t) \mathbf{A}_{1}\right] \boldsymbol{x} .
$$

We have assumed that the time dependency is simple enough that we can write it as $\epsilon \eta(t) \mathbf{A}_{1}$ where $\epsilon$ is a small parameter, $\mathbf{A}_{1}$ is a constant matrix that may depend on the basic state and $\eta(t)$ determines the nature of the time variations. The two extreme examples are where $\eta(t)$ is periodic or stochastic.

In the special case where $\mathbf{A}_{0}$ and $\mathbf{A}_{1}$ commute, we can write down the exact solution for any matrices as

$\boldsymbol{x}=e^{\mathbf{A}_{0} t+\epsilon \mathbf{A}_{1} \int_{0}^{t} \eta(s) d s} \boldsymbol{x}_{0}$.

where $\boldsymbol{x}_{0}$ is the initial condition. If moreover we assume that $\eta(t)$ is periodic with zero mean then the stability of the above system is determined entirely by $\mathbf{A}_{0}$. This is because we can apply Floquet analysis to this solution, and this dictates that we only need to determine how the system has grown after one period. Since we assume that $\eta(t)$ has zero mean, the $\mathbf{A}_{1}$ matrix makes no contribution. This scenario describes a broad range of problems where the stability of the average is precisely the same as the stability of the time-dependent state. Even though this may occur this is by no means necessary, as we shall soon see.

We can conclude that if $\eta(t)$ is periodic with zero mean, which we can take without lose of generality by simply redefining the two matrices, it is necessary that the two matrices do not commute in order to get PR.

\section{Stability of stochastic systems}

Consider the non-autonomous system from the previous section where $\eta(t)$ still has zero mean but now where it is a Gaussian stochastic variable. It has been stated in Farrell and Ioannou (1996b) that for PI to occur it is necessary that the two matrices do not commute. However, in this section we will illustrate why this is not the case and that PI can occur in stochastic systems even if the matrices commute.

The argument that Farrell and Ioannou (1996b) put forward is that the noise term is smaller than time, in particular

$\int_{0}^{t} \eta(s) d s \approx \sqrt{t}$.

A statement which relies on fact that the noise is white. Since exponential growth needs to be $O(t)$ they deduce that the stochastic noise cannot contribute exponential behaviour.

To determine the validity of their deduction consider the non-autonomous equation from the previous section with the added properties that the noise is Gaussian, red, and has the following statistics

$\langle\eta(t)\rangle=0 \quad$ and $\quad\langle\eta(t) \eta(t-\tau)\rangle=\alpha e^{-|\tau| / \tau_{c}}$,

where $\tau_{c}$ is the correlation time for the noise and $\alpha$ is the variance. We can recover the white noise limit by taking $\tau_{c} \rightarrow 0$ while keeping $\alpha \tau_{c}$ constant. The white noise limit is a mathematical idealisation that cannot be physically realisable since all random physical situations will have a nonzero correlation time, no matter how small. Nevertheless, white noise is a useful concept which is why we consider the limit of vanishing correlation time. 
Multiplying the non-autonomous Eq. (1) by the integrating factor $e^{-\mathbf{A}_{0} t}$ and defining $\boldsymbol{q}=e^{-\mathbf{A}_{0} t} \boldsymbol{x}$, allows the governing equation to be rewritten as

$\frac{d \boldsymbol{q}}{d t}=\epsilon \eta(t) e^{-\mathbf{A}_{0} t} \mathbf{A}_{1}(t) e^{\mathbf{A}_{0} t} \boldsymbol{q} \equiv \epsilon \eta(t) \mathbf{M}(t) \boldsymbol{q}$.

The solution to this equation can be written as

$\boldsymbol{q}(t)=\boldsymbol{q}_{0}+\epsilon \int_{0}^{t} \eta\left(t^{\prime}\right) \mathbf{M}\left(t^{\prime}\right) \boldsymbol{q}\left(t^{\prime}\right) d t^{\prime}$,

where we have introduced the following notation

$\mathbf{M}(t) \equiv e^{-\mathbf{A}_{0} t} \mathbf{A}_{1} e^{\mathbf{A}_{0} t}$.

This solution is stochastic since it is defined in terms of the random function $\eta(t)$. Solving this equation is difficult and may not be of general interest. While this equation is difficult to solve, solutions for the mean, the variance, and further higher order statistics can be found by defining the ensemble mean over different solutions to the equations, denoted by \langle\rangle as already used in Eq. (2).

The solution for $\boldsymbol{q}$ is implicit since the solution appears under the integral sign. One way to obtain an approximate solution is to iteratively substitute the equation into itself, take the mean of the resulting equation to obtain the following integral equation

$$
\begin{aligned}
& \langle\boldsymbol{q}\rangle=\boldsymbol{q}_{0}+ \\
& \epsilon^{2} \int_{0}^{t} \int_{0}^{t^{\prime}} \mathbf{M}\left(t^{\prime}\right) \mathbf{M}\left(t^{\prime \prime}\right)\left\langle\eta\left(t^{\prime}\right) \eta\left(t^{\prime \prime}\right) \boldsymbol{q}\left(t^{\prime \prime}\right)\right\rangle d t^{\prime \prime} d t^{\prime} .
\end{aligned}
$$

An exact solution is generally not possible as it requires computing the expectation of a cubic quantity which in turn contains the sought solution. As an alternative, we decided to approximate the equation based upon two physical assumptions. First, observe from the ODE governing $\boldsymbol{q}$ that the solution varies on a time scale proportional to $1 / \epsilon$, which is long since $\epsilon<<1$, by assumption. In addition, we assume that the correlation time is short compared to this time scale so that

$\tau_{c}<<\frac{1}{\epsilon}$.

As a result, the correlation function varies on a short time scale and is nearly zero whenever $t^{\prime}$ and $t^{\prime \prime}$ differ by more than $\tau_{c}$. Since the solution varies on a time scale longer than this we can replace the appearance of $\boldsymbol{q}\left(t^{\prime \prime}\right)$ by $\langle\boldsymbol{q}\rangle$ in the expectation of the cubic

$\left\langle\eta\left(t^{\prime}\right) \eta\left(t^{\prime \prime}\right) \boldsymbol{q}\left(t^{\prime \prime}\right)\right\rangle \approx\left\langle\eta\left(t^{\prime}\right) \eta\left(t^{\prime \prime}\right)\right\rangle\langle\boldsymbol{q}\rangle$.

Next we differentiate (Eq. 3) with respect to time to obtain,

$\frac{d}{d t}\langle\boldsymbol{q}\rangle=\epsilon^{2} \int_{0}^{t} M(t) M\left(t^{\prime \prime}\right)\left\langle\eta(t) \eta\left(t^{\prime \prime}\right)\right\rangle d t^{\prime \prime}\langle\boldsymbol{q}\rangle$.

We can rewrite the integral equation with respect to the variable $\langle\boldsymbol{x}\rangle$ and then approximate the integral to go to infinity. The error introduced is on the order of the approximation we have already made (the interested reader is directed to
Van Kampen, 2001, Chapter 16 page 400 for further details). Finally, the resulting equation is what is referred to as the Bourret Integral Equation

$\frac{d}{d t}\langle\boldsymbol{x}\rangle=\left\{\mathbf{A}_{0}+\right.$

$\left.\epsilon^{2} \int_{0}^{\infty}\langle\eta(t) \eta(t-\tau)\rangle \mathbf{A}_{1} e^{\mathbf{A}_{0} \tau} \mathbf{A}_{1} e^{-\mathbf{A}_{0} \tau} d \tau\right\}\langle\boldsymbol{x}\rangle$.

The effect of the noise is very small since the effect of the stochasticity in the above equation is second order in $\epsilon$. However, this still gives us a quantitative measure of the effect of stochastic variation.

Considering the case where $\mathbf{A}_{0}$ and $\mathbf{A}_{1}$ commute, the expression simplifies to

$$
\begin{aligned}
\frac{d}{d t}\langle\boldsymbol{x}\rangle & =\left\{\mathbf{A}_{0}+\epsilon^{2} \mathbf{A}_{1}^{2} \int_{0}^{\infty}\langle\eta(t) \eta(t-\tau)\rangle d \tau\right\}\langle\boldsymbol{x}\rangle \\
& =\left\{\mathbf{A}_{0}+\epsilon^{2} \mathbf{A}_{1}^{2} \alpha \tau_{c}\right\}\langle\boldsymbol{x}\rangle,
\end{aligned}
$$

where we have used the autocorrelation of coloured noise, Eq. (2), in the integral. The white noise limit is

$\frac{d}{d t}\langle\boldsymbol{x}\rangle=\left\{\mathbf{A}_{0}+\epsilon^{2} \mathbf{A}_{1}^{2} \alpha\right\}\langle\boldsymbol{x}\rangle$

Observe that both white and coloured noise can affect the solution since in general the second term in the parentheses need not be zero. Therefore, even if $\mathbf{A}_{0}$ and $\mathbf{A}_{1}$ commute, the stability of a stochastic system need not be the same as the stability of its time averaged mean. We will consider a special case in a subsequent section to illustrate this point.

\section{Stochastic harmonic oscillator}

Perhaps the simplest model to test the Bourret approximation is the simple harmonic oscillator. The extension we consider is a modification of the Mathieu equation where the natural frequency of the pendulum is not a periodic function of time but instead a stochastic function of time. This problem can also be found in Van Kampen (2001).

The equation, in nondimensional form, is

$\frac{d^{2} y}{d t^{2}}+(1+\epsilon \eta(t)) y=0$.

The $\epsilon$ in front of the stochastic variable $\eta(t)$ indicates that the variation from the mean frequency is slight. This equation can rather straightforwardly be written as a system of ODEs. Instead of doing so we will instead keep the equation in the above form since it is easier to interpret. By applying the Bourret approximation we deduce that to first order in $\epsilon^{2}$, the governing equation for the mean of the stochastic solution $\langle y\rangle$ is

$$
\begin{gathered}
\frac{d^{2}}{d t^{2}}\langle y\rangle+\left(\frac{2 \epsilon^{2} \alpha \tau_{c}^{3}}{1+4 \tau_{c}^{2}}\right) \frac{d}{d t}\langle y\rangle \\
+\left(1-\frac{\epsilon^{2} \alpha \tau_{c}^{2}}{1+4 \tau_{c}^{2}}\right)\langle y\rangle=0 .
\end{gathered}
$$




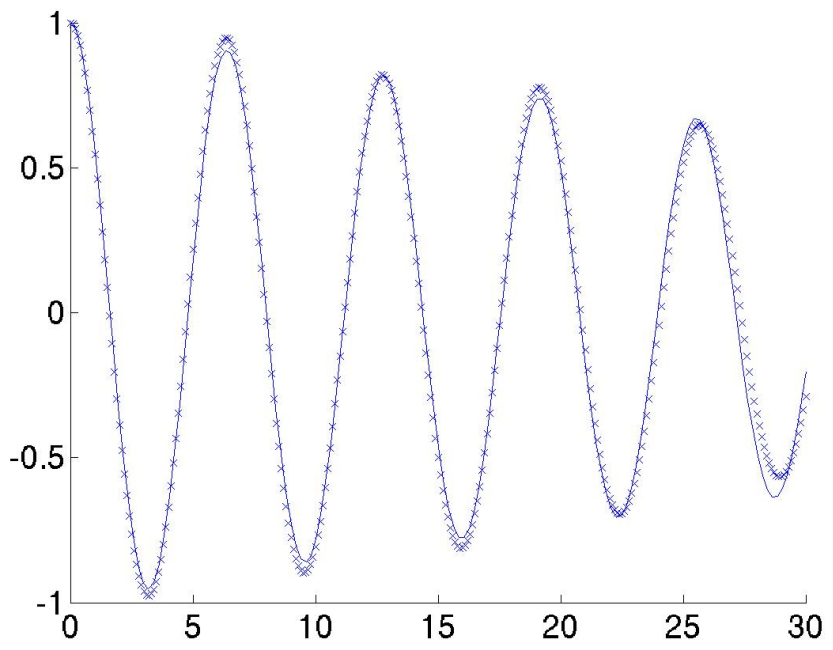

Fig. 1. Comparison of the Bourret solution (solid line) with the Monte Carlo solution (crosses) of 500 ensemble members for the stochastic harmonic oscillator with $\alpha=0.5$ and $\tau_{c}=0.5$.

Note that even though the influence of the stochastic coloured noise is slight, the effect is two-fold. First it modifies the natural frequency of the mean solution, where the frequency is necessarily reduced. The second impact is to introduce a damping term into the equation. Even though the damping is slight, the decay time scale is on the order of $\left(1+4 \tau_{c}^{2}\right) /\left(2 \epsilon^{2} \alpha \tau_{c}^{3}\right)$. To take the white noise limit we write $\alpha=\alpha / \tau_{c}$, and then take the limit as $\tau_{c} \rightarrow 0$ (Van Kampen, 2001). In doing this the damping term tends to zero as does the modification to the frequency. This demonstrates that white noise does not alter the behaviour of the mean. For the noise to alter the mean we must have a nonzero correlation time. As stated before, physical problems inherently have a nonzero correlation time so the coloured noise is the case of greater interest. However, the fact that we are restricted to short correlation times is limiting. To explore the case of order one correlation times it is necessary to consider Monte Carlo simulations.

The solution of Eq. (5) yields approximately the same answer as Monte Carlo solutions to Eq. (4). To obtain the latter we simulate many solutions to the stochastic ODE Eq. (4) using an Euler method and Gaussian random numbers of the specified mean and variance. Then we ensemble average over all of these solutions to obtain $\langle y\rangle$. As the number of members in the ensemble increases the error between our two solutions must decrease. Figure 1 plots the two solutions for the special case of $\alpha=0.5$ and $\tau_{c}=0.5$. We readily observe that there is a close correspondence between the Bourret solution, denoted by the solid line, and the Monte Carlo solution, denoted by the crosses. Observe also that the solution decays as is predicted by the theory.

Figure 2 compares the Bourret solution with the Monte Carlo solution for the same choice of parameters except where the correlation time is larger, $\tau_{c}=0.75$, and hence the damping is stronger. There is also a greater discrepancy with the frequency, though this is more difficult to observe from the figure.

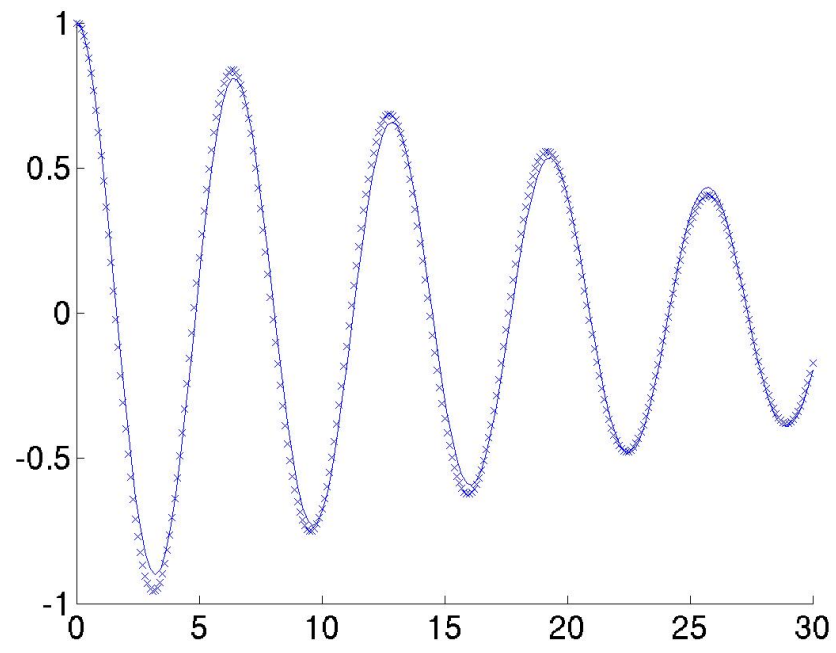

Fig. 2. Comparison of the Bourret solution (solid line) with the Monte Carlo solution (crosses) of 2000 ensemble members for the stochastic harmonic oscillator with $\alpha=0.5$ and $\tau_{c}=0.75$.

The result that the ensemble mean of the harmonic oscillator with random frequency decays in time is true but must be carefully interpreted. This does not imply that typically each member of the ensemble decays since it does not, as is readily observed from the fact that the governing equation for the stochastic variable does not have a damping term. Each solution is initialised with the same initial conditions but they grow out of phase as time evolves due to the differing frequencies. Averaging over these out of phase solutions is what is responsible for the decay in the ensemble mean. This is why asymptotic stability in the ensemble mean does not imply asymptotic stability in each stochastic realisation, even in an averaged sense. However, if the ensemble mean is unstable that does necessarily imply that on average, each member of the ensemble is unstable.

\section{Stochastic barotropic instability}

The next problem we study is a natural extension of the time-periodic barotropic shear flow discussed in Poulin et al. (2003). The model we are considering is the twodimensional vorticity equation (also equivalent to the quasigeostrophic equation) for a homogeneous inviscid fluid with a flat bottom and a rigid lid (Pedlosky, 1987). The horizontal shear flow is divided into three strips of spatially uniform vorticity. The two strips at each end are semi-infinite and have fixed vorticity but the center strip has a vorticity that can vary in time. The velocity fields are chosen so that the velocity is continuous at the interfaces. This problem is similar to those studied using contour dynamics and consequently we need only determine how the two interfaces between the three regions evolve in time. The interested reader is directed to Poulin et al. (2003) for further details of the model.

If we denote the vorticity at the interface by the 2 -vector $\boldsymbol{q}$ then the linearised equation for the perturbation vorticity can 


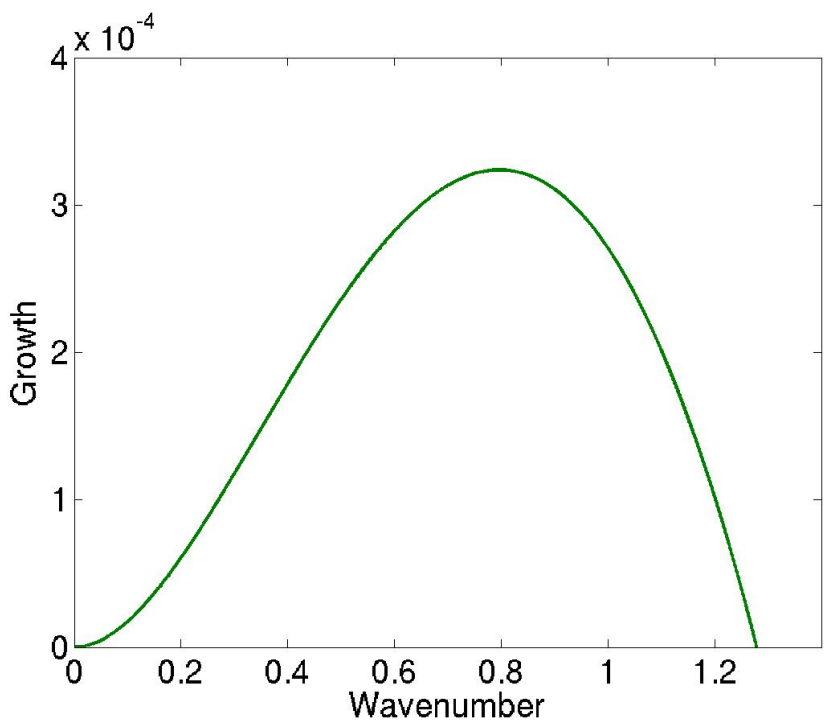

Fig. 3. Growth rate against wave number for the stochastic barotropic shear flow with zero mean vorticity obtained from Eq. (5).

be written as

$\frac{d \boldsymbol{q}}{d t}=\left[\mathbf{A}_{0}+\epsilon \eta(t) \mathbf{A}_{1}\right] \boldsymbol{q}$,

where the two matrices are defined by

$\mathbf{A}_{0}=\frac{i}{2} q_{0}\left[\begin{array}{cc}-1 & 0 \\ 0 & 1\end{array}\right]$,

$\mathbf{A}_{1}=\frac{i}{2} \epsilon \eta(t)\left[\begin{array}{cc}(k-1) & -\exp (-k) \\ \exp (-k) & -(k-1)\end{array}\right]$

The variables that appear are

$$
\begin{array}{cll}
\mathrm{k} & - & \text { wavenumber in the direction of flow } \\
q_{0} & - & \text { mean vorticity of the shear } \\
\epsilon \eta(t) & - & \text { difference from the mean vorticity } \\
\epsilon & - & \text { square root of the variance of the stochasticity } \\
\eta(t) & - & \text { stochastic variable }
\end{array}
$$

The Bourret approximation can be applied to obtain the following governing equation for the mean of the stochastic solution

$$
\frac{d}{d t}\langle\boldsymbol{q}\rangle=\left[\mathbf{A}_{0}+\overline{\mathbf{A}}_{1}\right]\langle\boldsymbol{q}\rangle
$$

where the constant matrix $\overline{\mathbf{A}}_{1}$ arising from the fluctuations is

$\overline{\mathbf{A}}_{1}=\left(\frac{\epsilon}{2}\right)^{2} \alpha \tau_{c}\left[\begin{array}{ll}a_{11} & a_{12} \\ a_{21} & a_{22}\end{array}\right]$,

with

$$
\begin{aligned}
& a_{11}=\frac{e^{-2 k}}{1-i k q_{0} \tau_{c}}-(k-1)^{2}, \\
& a_{12}=-(k-1) e^{-k} \frac{i k q_{0} \tau_{c}}{1+i k q_{0} \tau_{c}},
\end{aligned}
$$

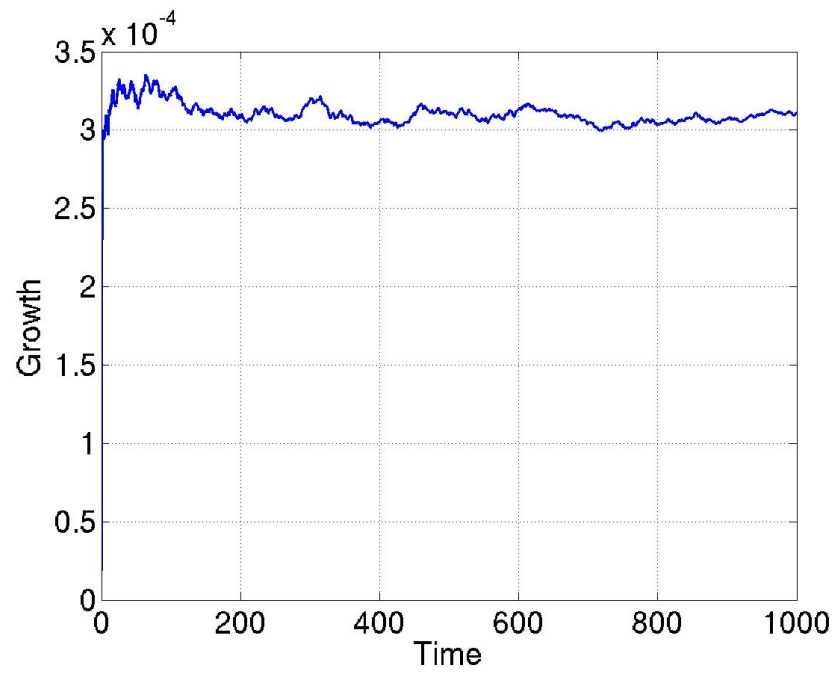

Fig. 4. Growth rate against time for wave number $k=0.8$ for the stochastic barotropic shear flow with zero mean vorticity obtained from Monte Carlo simulation with 1000 members.

$a_{21}=(k-1) e^{-k} \frac{i k q_{0} \tau_{c}}{1-i k q_{0} \tau_{c}}$,

$a_{22}=\frac{e^{-2 k}}{1+i k q_{0} \tau_{c}}-(k-1)^{2}$.

Since the above expression is linear in the correlation time we observe that, unlike in the stochastic harmonic oscillator, both coloured and white noise alter the behaviour of a barotropic shear flow.

The solution to the averaged equation can be readily obtained and then compared with the result of a Monte Carlo simulation of the original stochastic differential equation. The solution to the Bourret integral equation is illustrated in Fig. 3 for the special case of $q_{0}=0$; where the mean flow is zero and the average at the flow is stable. Even though the growth rates are very small we notice that there is a range of wavenumbers in which the growth rates are nonzero and hence the system is unstable. There is a high wavenumber cut off, but no low wavenumber cut off. The most unstable wavenumber is close to $k=0.8$ and corresponds to a growth rate of approximately $3.2 \times 10^{-4}$.

We have also computed a Monte Carlo simulation for $k=0.8$ for a case of one thousand members in the ensemble. The results are illustrated in Fig. 4. The growth rate for this wavenumber is $3.1 \times 10^{-4}$ which is comparable to the theory predicted from the Bourret approximation. By comparing this to another simulation with five hundred members we approximate the error to be smaller than $4 \times 10^{-5}$. There is a small difference between the two growth rates which arises from the fact that the analytical solution we obtain is only an approximate solution. In the limit as the variance of the random variable approaches zero we expect this error to also approach zero. 


\section{Conclusions}

It has previously been determined that time-periodic variation in shear flows can give qualitatively different predictions for the stability of a flow rather than just considering the time-average mean of the shear (Pedlosky and Thomson, 2003; Poulin et al., 2003). In this article we have explored the case in which the time dependence is completely stochastic, and the noise can either be uncorrelated (white noise) or correlated over a short correlation time (coloured noise). Instabilities that arise in stochastic shear flows are a natural extension of parametric resonance and hence we call this process stochastic parametric resonance.

We have considered the limit of slowly varying solutions with short correlation times and have derived a special case of the Bourret Integral equation, which is an approximate solution. Stochastic Parametric Resonance can occur even if the time averaged solution is stable. The Bourret approximation to the non-autonomous linear system indicates that even if the two matrices $\mathbf{A}_{0}$ and $\mathbf{A}_{1}$ commute resonance can still occur, contrary to what is suggested by Farrell and Ioannou (1996b). The particular model we studied was for a barotropic shear flow but we have also studied baroclinic shear flows and have found qualitatively similar results; stochastic variation can generate instability.

Some future areas of research are to explore a wider range of parameter space in order to learn about how the stochasticity affects the problems in general. Also, by using Monte Carlo solutions we can investigate the effect of having order one or larger correlation times. The Bourret solutions shows that the growth rates increase as the correlation times increase, however, we do not know whether this relationship persists beyond the range of validity of the Bourret solution: the correlation time of the noise is shorter than the time scale for variation of the solution. Also, studying the nonlinear evolution of stochastic parametric resonance is of great interest. The latter is under investigation by Flierl and Pedlosky (personal communication).
Acknowledgements. The authors would like to thank M. Stastna and G. R. Flierl for discussions that helped to elucidate some important ideas on stochastic processes. Also, we thank NSERC for financial support during the preparation of this manuscript.

Edited by: W.-G. Früh

Reviewed by: two referees

\section{References}

Farrell, B. and Ioannou, P.: Generalized stability theory Part I: autonomous operators, J. Atmos. Sci., 53, 2025-2040, 1996a.

Farrell, B. and Ioannou, P.: Generalized stability theory Part II: nonautonomous operators, J. Atmos. Sci., 53, 2041-2053, 1996 b.

Flierl, G.: Application of linear quasi-geostrophic dynamics to gulfstream rings, J. Phys. Oceanogr., 7, 443-453, 1977.

Griffiths, R., Killworth, P., and Stern, M.: Ageostrophic instability of ocean currrents, J. Fluid Mech., 117, 343-377, 1982.

Nayfeh, A. and Mook, D.: Nonlinear oscillations, Wiley interscience, 1995.

Pedlosky, J.: Geophysical Fluid Dynamics, Springer-Verlag, 1987.

Pedlosky, J. and Thomson, J.: Baroclinic instability of timedependent currents, J. Fluid Mech., 490, 189-215, 2003.

Poulin, F., Flierl, G., and Pedlosky, J.: The Instability of TimeDependent Shear Flows, J. Fluid Mech., 481, 329-353, 2003.

Stoker, J.: Nonlinear vibrations in mechanical and electrical systems, Interscience Publishers, 1950.

Van Kampen, N.: Stochastic processes in physics and chemistry, North Holland Personal Library, 2001. 